\title{
Hydrogen retention in beryllium: concentration effect and
}

\section{nanocrystalline growth}

C. Pardanaud ${ }^{*}, 1$, M. I. Rusu ${ }^{1}$, C. Martin ${ }^{1}$, G. Giacometti ${ }^{1}$, P. Roubin ${ }^{1}$, Y. Ferro ${ }^{1}$, A. Allouche ${ }^{1}$, M. Oberkofler $^{2}$, M. Köppen ${ }^{3}$, T. Dittmar ${ }^{3}$, Ch. Linsmeier ${ }^{3}$

${ }^{1}$ Laboratoire PIIM, Aix-Marseille Université/CNRS, Avenue escadrille Normandie-Niemen, 13397, Marseille

${ }^{2}$ Max-Planck-Institut für Plasmaphysik, Boltzmannstraße 2, D-85748 Garching, Germany

${ }^{3}$ Forschungszentrum Jülich $\mathrm{GmbH}$, Institut für Energie- und Klimaforschung - Plasmaphysik, 52425 Jülich, Germany

Keywords: Hydrogen storage, plasma-wall interaction, Raman microscopy, beryllium hydride, crystalline growth

* Corresponding author: e-mail cedric.pardanaud@univ-amu.fr ,Phone: +33491282707 


\section{Abstract}

We herein report on the formation of $\mathrm{BeD}_{2}$ nanocrystalline domes on the surface of a beryllium sample exposed to energetic deuterium ions revealing a potential of storing hydrogen. A polycrystalline beryllium sample was exposed to $D$ ions at $2 \mathrm{keV} /$ atom leading to laterally averaged deuterium areal densities up to $3.510^{17} \mathrm{D} \mathrm{cm}^{-2}$, and studied using nuclear reaction analysis, Raman microscopy, atomic force microscopy, optical microscopy and quantum calculations. Incorporating D in beryllium generates a tensile stress that reaches a plateau at $\approx 1.510^{17} \mathrm{D} \mathrm{cm}^{-2}$. For values higher than $2.010^{17} \mathrm{~cm}^{-2}$, we observe the growth of $\approx 90 \mathrm{~nm}$ high dendrites, which cover up to $10 \%$ of the surface in some zones of the sample when the deuterium concentration is $3 \times 10^{17} \mathrm{D} \mathrm{cm}^{-2}$. These dendrites are associated to the Raman spectrum of crystalline $\mathrm{BeD}_{2}$. They are candidates to explain low temperature thermal desorption spectroscopy peaks observed when bombarding Be samples with $\mathrm{D}$ ions with fluencies higher than $1.210^{17} \mathrm{D} \mathrm{cm}^{-2}$. This crystal growth observed on the top of a bulk material may be used in theory to produce nanometric powders that could be used in principle for storing hydrogen efficiently. 


\section{Introduction}

The problematic of hydrogen isotope retention in beryllium addresses main issues related to plasma wall interactions in tokamaks [1] and to hydrogen storage [2].

Plasma wall interactions (erosion, retention, material modification, dust formation,...) occurring in tokamaks like the future ITER reactor (devoted to demonstrate the feasibility of obtaining energy from a magnetically confined $D+T$ plasma) can lead to tritium retention, which is a major safety issue, due to its radioactivity $[3,4]$. Hydrogen isotope retention in beryllium can also play a role in the life time estimation of the $\approx 700 \mathrm{~m}^{2}$ ITER's beryllium inner walls because of erosion [5]. This explains why several studies based on ion irradiation of Be samples have been devoted to $D$ behavior to ensure that tritium retention will not be a limiting issue in ITER operations. For deuterium kinetic energies in the range $15-60 \mathrm{eV}$, a D/Be ratio up to 0.7 has been reached in forming co-deposited Be layers and by varying substrate temperature and deposition rate [6, 7]. Older data also focusing on co-deposits reported values in the range $\mathrm{D} / \mathrm{Be}=0.1-0.4[8-10]$. Values in the range $\mathrm{D} / \mathrm{Be}=0.2-0.4$ have been measured under ion implantation of $20 \mathrm{keV} / \mathrm{D}$ with $10^{19} \mathrm{D} \mathrm{cm}^{-2}$ [11]. For 9 keV/D implantation, a storage of $D$ as $D_{2}$ molecules has been reported in bubbles tending to interconnect in the material [12]. Studies of 0.6 and $1 \mathrm{keV} / \mathrm{D}$ ions impinging on polycrystalline beryllium samples have shown that at fluencies $\approx 210^{17} \mathrm{~cm}^{-2}$, there is a saturation of the $D$ content with a $\mathrm{D} / \mathrm{Be}$ ratio in the range $0.2-0.3[13,14]$, close to values reported in [15]. The diversity of results given above indicates impinging energies are not the only parameter driving the $\mathrm{D} / \mathrm{Be}$ ratio. The thermal desorption spectroscopy of such samples displayed a single $D_{2}$ peak at $900 \mathrm{~K}$ for fluences lower than $0.710^{17} \mathrm{~cm}^{-2}$. A second peak was shown to appear at $750 \mathrm{~K}$ at a fluence of $\approx 110^{17} \mathrm{~cm}^{-2}$. Two additional peaks appeared between 450 and $500 \mathrm{~K}$ for fluences higher than $1.210^{17} \mathrm{~cm}^{-2}$, a structural modification being involved to explain their existence. A comparative study of thermal desorption of $\mathrm{D}$ implanted in Be samples with two different crystalline orientation and in a polycrystalline Be sample has been done, revealing the role of both crystal orientation and D diffusion along grain boundaries under thermal annealing [16]. It should be noted that none of all 
these studies have reported the formation of beryllium hydride in the form $\mathrm{BeH}_{2}$, except in [14] where it was suggested.

To act efficiently as an energy carrier, hydrogen should be absorbed and desorbed easily in specific materials, developed to fit with these two constraints $[17,18] . \mathrm{MgH}_{2}$ is the prototype of the metallic hydrides having an interesting gravimetric capacity (7.6 wt.\%), but its decomposition temperature is too high compared to others [19]. Beryllium hydride, $\mathrm{BeH}_{2}$ being one of them, has been envisaged very recently, from a theoretical point of view, as a promising and more efficient hydrogen storage media than $\mathrm{MgH}_{2}$ [20]. It has been synthesized in the past in a crystalline form [21, 22], in an amorphous form [23], and transitions between crystalline and amorphous forms have been studied [24]

In this study we report on the formation of $\mathrm{BeD}_{2}$ crystalline structures (i.e. $\mathrm{D} / \mathrm{Be}=2$ ) by using plasma irradiation, and on their characterization by using nuclear reaction analysis (NRA), atomic force microscopy (AFM), optical microscopy, and quantum calculations compared to Raman microscopy. It was shown previously that this latter technique is sensitive to the way hydrogen isotopes are bonded and organized in materials and thus that it can be relevant for both fusion [25] and hydrogen storage [26] applications. This work is organized as follows: experimental and computational details are given in Section 2. Results obtained from the computational study are presented in Section 3. Results obtained from experimental measurements are presented in Section 4. A discussion highlighting the consistency of all these results is presented in Section 5. Conclusions are given in Section 6. 


\section{Experimental and computational details}

\subsection{Ion implantation and sample characterization}

The experimental study focuses on a $1.5 \mathrm{~cm}$ diameter polycrystalline beryllium sample that has been bombarded by a relatively homogeneous beam of $6 \mathrm{keV} \mathrm{D}^{3+}(2 \mathrm{keV}$ per deuterium) at a fluence of $1.910^{17} \mathrm{D} \mathrm{cm}^{-2}$. At the center of the sample, roughly $30 \%$ of the samples surface contains $1.010^{17}$ deuterium atoms. On the other parts of the sample, the number of implanted deuterium atoms decreases rapidly down to $\approx 0$ at the edges. Sample implantation and in situ ion-beam analysis are performed in the ultra high vacuum experiment ARTOSS [27]. Detailed information on experimental setup, sample and procedure are published elsewhere [28]. In brief, a polycrystalline Be sample is cleaned by cyclic sputtering with $\mathrm{Ar}^{+}$ions and subsequent annealing to $1000 \mathrm{~K}$. This sample is exposed to a mass separated beam of $\mathrm{D}_{3}{ }^{+}$ions at $2 \mathrm{keV} / \mathrm{D}$ with a fluence of $1.9 * 10^{17} \mathrm{D} \mathrm{cm}{ }^{-2}$ in the central area of $5^{*} 8 \mathrm{~mm}^{2}$. The amount of deuterium trapped is measured on several locations of the sample surface by means of the $D\left({ }^{3} \mathrm{He}, \mathrm{p}\right) \mathrm{He}$ nuclear reaction with a lateral resolution on the order of $1 \mathrm{~mm}$. Raman spectra were also recorded in the vicinity of some of these regions. The numbers given in that figure, and referred to as $N_{D}$ later in the text and figures, are the absolute numbers of implanted $D$ atoms measured by means of this nuclear reaction. For comparison with results from the literature we give $\mathrm{N}_{\mathrm{D}}$ in deuterium per $\mathrm{cm}^{2}$.

Raman spectra were recorded on several locations of the sample, using a Horiba-Jobin-Yvon HR LabRAM apparatus (with $\times 100$ objective, with a numerical aperture of 0.9 ) in the backscattering geometry. $\lambda_{\mathrm{L}}=514.5$ and $632.8 \mathrm{~nm}$ were used. The laser power was kept at $\sim 1 \mathrm{~mW} \mu \mathrm{m}^{-2}$ to prevent damages. Spectra were recorded with various exposure times to check that samples do not evolve under laser irradiation. We also used, when indicated in the text, the Raman imaging mode. It consists in moving the sample under the laser beam by $0.5 \mu \mathrm{m}$ steps, and recording spectra at all the locations probed. Optical images obtained with the $100 x$ objective used for making Raman spectra were recorded on several locations and some images were thresholded and binarized to determine 
the surface coverage of structures detailed below. Atomic force microscopy (AFM) was performed on some zones of the sample using the tapping mode of an AFM-NTMDT solver. The tip radius was smaller than $10 \mathrm{~nm}$, the vertical and horizontal resolutions were $\approx 1 \mathrm{~nm}$ and $\approx 10 \mathrm{~nm}$, respectively.

\subsection{Computational details}

The calculations were performed within the framework of the spin-polarized gradient-corrected density functional theory (DFT) using the Perdew-Burke-Ernzerhof functionals (PBE [29]) and including a semi-empirical pairwise London damped dispersion correction (PBE-D2 [30, 31]). A plane-wave basis set was used with kinetic energy cutoff for wavefunctions of 70 Rydberg (952 eV). The calculations were carried out using the Quantum-Espresso suite [32]. The Vanderbilt ultrasoft pseudo potentials are taken from the GBRV database [33], all the electrons (core + valence) are included in the DFT calculation i.e. one $\mathrm{e}^{-}$for $\mathrm{H}$ and $4 \mathrm{e}^{-}$for $\mathrm{Be}$, this justifies the large energy cutoff. Within this methodology, the Be-H bond length of the $\mathrm{BeH}_{2}$ molecule is calculated to $1.340 \AA$, in good agreement with experimental data (1.332 to $1.342 \AA$ [34]); the vibrational frequencies are $v_{1}=707.8, v_{2}=1990.6$ and $v_{3}=2225.9 \mathrm{~cm}^{-1}$, in very good agreement with results based on many body Coupled Cluster CCSD(T) / ccpV6Z calculations [35], $v_{1}=713.5, v_{2}=1985.2 \mathrm{~cm}^{-1}$ and $v_{3}=2171.5 \mathrm{~cm}^{-1}$ and experimental results $v_{3}=2178.9 \mathrm{~cm}^{-1}$ [34]. These frequencies are plotted in Figure 1. The height of the peak is proportional to the number of modes and the peaks are convoluted by $20 \mathrm{~cm}^{-1}$ half-width Gaussian functions. Figures 2 to 4 are plotted the same way. As a consequence, these Figures cannot be directly compared to Raman spectrum , except for the bottom of figure 2 where selection rule are also taken into account.

The calculated lattice parameter of the hexagonal-compact unit-cell containing two beryllium atoms is $\mathbf{a}=2.28$. It is in very good agreement with the experimental one $2.29 \AA$, and in better agreement than previous DFT-GGA results [36] [37]. This improvement is the consequence of adding London dispersion forces; it also improves the bulk modulus determined to $120 \mathrm{GPa}$ (exp: 110 127 
GPa). The phonon spectrum at the $\Gamma$ point of the Brillouin zone is made of three frequencies 469.2 , 469.2 and $723.9 \mathrm{~cm}^{-1}$, with deviations lower than $7 \%$ as compared with experimental results [38].

Studying trapping of deuterium atoms in beryllium requires supercells to avoid interactions between the repeated images from cells to cells. The $2 \times 2 \times 2$ supercell ( $16 \mathrm{Be}$ atoms) is the compromise we choose between the size of the system and the computational cost required.

\section{Computational studies}

We first calculated the phonon spectra of a $\mathrm{BeD}_{2}$ molecule in a beryllium crystal. $\mathrm{BeD}_{2}$ was built by adding two deuterium atoms to a beryllium of the crystal network while keeping the cell parameters unchanged. Two configurations were considered for $\mathrm{BeD}_{2}$ : they are shown in inset of Figure 1 and will be referred as linear and bent.

The linear configuration is the most stable one. More precisely, the linear $\mathrm{BeD}_{2}$ geometry is such that $\theta_{\mathrm{D}-\mathrm{Be} \_\mathrm{D}}=169^{\circ}$ and the two non-equivalent Be-D distances are $1.44 \AA$ and $1.51 \AA$. The phonon spectrum of the linear $\mathrm{BeD}_{2}$ is shown in Figure 1 ; it is markedly different from that of the molecule in the gas phase. The bent configuration is $0.1 \mathrm{eV}$ less stable than the linear one. The geometry is such

that $\theta_{\mathrm{D}-\mathrm{Be} \_\mathrm{D}}=71^{\circ}$ and the non-equivalent Be-D distances are $1.51 \AA$ and $1.61 \AA$. The phonon spectrum is shown in Figure 1. It's specific feature is to exhibit two low frequencies at 627 and $653 \mathrm{~cm}^{-1}$, which would make the bent configuration in principle identifiable by Raman measurements.

The lattice system of crystalline beryllium hydride is orthorhombic. The beryllium atoms are in a tetrahedral-like symmetry; their environment is described in Figure 3 where angles and bond-lengths distributions are plotted. Figure 3 reveals a single bond length at $1.41 \AA$ and two specific angles at $96^{\circ}$ and $116^{\circ}$ not so far from the $109^{\circ}$ of a perfect tetrahedron. The phonon spectrum of the crystalline Beryllium hydride $\left(\mathrm{BeD}_{2}\right)$ is shown in the lower part of Figure 2. It is computed taking into account selection rules for Raman spectroscopy. As a consequence, this spectrum is directly comparable with Raman measurements. It is characterized by low frequencies components appearing below $500 \mathrm{~cm}^{-1}$, which again would make beryllium hydride experimentally identifiable. 
Finally, and in order to rationalized phonon spectra, we increased the D concentration starting from one $\mathrm{BeD}_{2}$ linear molecule in the $2 \times 2 \times 2$ supercell $(\mathrm{D} / \mathrm{Be}=0.125)$ to the full $\mathrm{D} / \mathrm{Be}=2$ ratio maintaining the hexagonal symmetry and keeping cell parameters frozen. As the D concentration increases, the angle and bond-lengths distributions come closer and closer to that of beryllium hydride (Figure 3) while the linear geometry is lost. This result tends to show that beryllium adopts a tetrahedral-like environment as the $\mathrm{D} / \mathrm{Be}$ ratio increases. The calculated phonon spectra (Figure 2) indicate that increasing the $D$ concentration produces the progressive enlargement of the spectrum with a shift toward the high frequencies, while the low frequencies remains more or less unchanged. However, the symmetry of crystalline beryllium hydride is orthorhombic, and the inter-beryllium atoms distance is different as compared to the hexagonal previous case. Such a difference induces a high stress in the hexagonal structure. We therefore relaxed the cell parameters of the hexagonal structure for two $\mathrm{D} / \mathrm{Be}$ ratios ( 0.5 and 1$)$ within the standard thermodynamic conditions ( $\mathrm{P}=1$ bar). This relaxation has the effect to make appear low frequency in the phonon spectrum as can be seen in Figure 4. As a conclusion, high frequencies without low frequencies in spectra must be considered as the signature of highly constrained structures. On the contrary, low frequencies along with the full spectrum we calculated make the crystalline beryllium hydride identifiable by Raman spectroscopy.

\section{Experimental studies}

The influence of the number of implanted $D$ atoms on the pristine material can be seen in Figure 5-a, which displays the spectral window where the beryllium stretching mode lies. The band, at $459 \mathrm{~cm}^{-1}$ for the pristine sample, downshifts and broadens when $N_{D}$ increases. This is usually interpreted as due to the creation of defects in the material induced by ion implantation. However, as it is known that Be-D bonds can be formed easily when D is implanted into a beryllium crystal, and that this results in a volume increase per beryllium atom [37], one can conclude, according to [39], that this downshift is induced by tensile stress in the material, exactly what is expected theoretically 
(see part III of this work). Note that a study of the effect of external compressive stress on a beryllium crystal has reported the upshift and the broadening of the band related to the beryllium stretching mode with the pressure increase [40]. Figure 5-b displays the $200-1800 \mathrm{~cm}^{-1}$ spectral window, with a zoom on the vertical axis. Contrary to what is expected by the Raman selection rules of a perfect Be crystal, which predict only the beryllium stretching mode to be active (found at 459 $\mathrm{cm}^{-1}$ in our study), the spectrum of the pristine sample is not flat elsewhere, especially in the 750 $1100 \mathrm{~cm}^{-1}$ region where large bumps are clearly seen. We do not want to interpret in details their origin here but we can notice that it is not related to the presence of $D$ in the material, as they are also displayed in the $D$ free spectrum, nor to the presence of a surface oxide layer because it does not fit with the 338,684, $1082 \mathrm{~cm}^{-1}$ frequencies reported for BeO [41]. When increasing $N_{D}$, several bands appear at $255,278,617,907,1397, \approx 1420,1696,1724 \mathrm{~cm}^{-1}$. Most of these bands have their wavenumbers close to the one calculated for the $\mathrm{BeD}_{2}$ crystal. It proves first that these bands are due to a Be-D bond, second: the $\mathrm{D} / \mathrm{Be}$ ratio should be close to 2 , and third: the material probed is crystalline, as the experimental and calculated spectra are very similar. Another argument suggests that it is crystalline: the full-widths-at-half-maximum (FWHM) of the Raman bands are close to 10 $\mathrm{cm}^{-1}$, contrary to what is generally measured for amorphous beryllium hydride (generally hundreds of $\mathrm{cm}^{-1}$ ) [42]. The intensities of these bands seem to be related to the amount of $D$ trapped in the material as they increase with $\mathrm{N}_{\mathrm{D}}$.

Having a crystalline structure subsequently to a high fluence implantation is counterintuitive and needs to have a closer insight. Figure 6 shows a survey of the surface morphology at different locations of the sample, obtained by means of optical microscopy. In that figure we display some images showing that in zones where $N_{D}<1.510^{17} \mathrm{D} \mathrm{cm}^{-2}$, some dark spots can be evidenced, their concentration depending on $N_{D}$. In zones where $N_{D}>1.510^{17} \mathrm{D} \mathrm{cm}^{-2}$ dendrites appear together with the already existing dark spots. The dendrite shape, contrast, size and concentration vary depending on $N_{D}$, too. For example, low contrast dendrites in Figure 6-d seems to be composed of disconnected 
1-2 $\mu \mathrm{m}$ size branches, whereas high contrast dendrites in Figure 6-g are composed of 5 (or more) $\mu \mathrm{m}$ size interconnected branches. Figures 7-a and 7-b show height measurements of these structures, such as those found in Figures 6-d and 6-g, by means of AFM. It appears more clearly than in the optical microscope images that the dendrites coexist with circular domes, the latter being the dark spots visible in Figure 6. From that we can conclude that domes appear at lower deuterium $N_{D}$ than dendrites. Dendrite heights are in the range $50-200 \mathrm{~nm}$, whereas domes are smaller. These two kinds of structures are clearly distinguishable in Figure 7-a, where dendrites appear dense and bulky and domes are regularly distributed on the surface. These two kinds of structures are not so clearly distinguishable in Figure 7-b where dendrites appear sparsely connected, and domes are not distributed regularly, as if they were constituting units of the nascent dendrites.

Figure 8 shows a closer look on the domes (Figure 8-a) and dendrites (Figure 8-b) by means of AFM. The domes are smoother than the dendrites, the latter can even be composed of two collinear branches. This observation suggests that dendrites are more complex objects than domes. Figure 8-c and 8-d display some dome and dendrite profiles, respectively. The larger heights can be as high as 75 and $160 \mathrm{~nm}$ for domes and dendrites, respectively. The dome average profiles exhibit a parabolic average shape with a $50 \mathrm{~nm}$ height and $600 \mathrm{~nm}$ bases. The average profiles of the dendrites are more rectangular with a $90 \mathrm{~nm}$ height and a $1 \mu \mathrm{m}$ base. The rectangularity is just the consequence of summing up single and double dendrite branches, and do not reflect the shape of single dendrite profiles, but it is useful for mean height estimation on all the sample.

Figure 9 shows Raman imaging in the vicinity of a dendrite, in the zone of the sample where $N_{D}=310^{17} \mathrm{D} \mathrm{cm}^{-2}$. In Figures 9-c and 9-d the intensities of the band close to $459 \mathrm{~cm}^{-1}$ due to the Be stretching mode, and the band at $1397 \mathrm{~cm}^{-1}$ due to a Be-D mode are displayed, respectively. These two bands are more intense on the dendrite than off the dendrite. In Figure 9-e, we compare the spectra on and off dendrite. These two spectra have been obtained by summing all the spectra over the two areas and normalizing to the band at $459 \mathrm{~cm}^{-1}$, the threshold distinguishing between on and off dendrite being fixed at a value of 100 in the Figure 9-c map. The 5 bands identified in Figure 5-b 
(and pointed by a star in Figure 9) as due to Be-D modes in a $\mathrm{BeD}_{2}$ crystal are much more intense on dendrites than off dendrites. The spatial correlation between the dendrites and the $\mathrm{BeD}_{2}$ Raman signature allows us to conclude that the dendrites, formed under ion bombardment, are a high reservoir of trapped deuterium.

Figure 10 displays the evolution with $\mathrm{N}_{\mathrm{D}}$ of two spectroscopic parameters related to the $\mathrm{Be}$ stretching mode (wavenumber, $v$, and FWHM) and compared to the dendrite surface coverage (obtained by thresholding + binarizing $\approx 4500 \mu \mathrm{m}^{2}$ optical images, as shown in the Figure $10-\mathrm{c}$ insert). From 0 to $1.510^{17} \mathrm{D} \mathrm{cm}^{-2}, v$ decreases linearly by $\approx 3 \mathrm{~cm}^{-1}$ and the FWHM increases linearly by $\approx 6 \mathrm{~cm}^{-}$

${ }^{1}$, for both laser wavelengths used. In this interval, the dendrite coverage is close to zero, meaning that most of $D$ atoms are implanted in $a \approx 50 \mathrm{~nm}$ depth layer, according to the implantation depth profile calculated using SRIM [43] (given in the insert of Figure 10), a free access Monte Carlo computer program based on the binary collision approximation. For $N_{D}=1.510^{17} \mathrm{D} \mathrm{cm}^{-2}$, and taking into account the $\approx 50 \mathrm{~nm}$ of implantation depth, it gives a value $\mathrm{D} / \mathrm{Be} \approx 0.24$, which is compatible with what was previously measured [14]. For $\mathrm{N}_{\mathrm{D}}>1.510^{17} \mathrm{D} \mathrm{cm}^{-2}, \mathrm{v}$ and FWHM both reach a plateau. The dendrite coverage starts to increase linearly from $\approx 0$ at $N_{D} \approx 210^{17} \mathrm{D} \mathrm{cm}^{-2}$ and reaches up to $10 \%$ close to $310^{17} \mathrm{D} \mathrm{cm}^{-2}$, without tendency for saturation in our experimental conditions. Note that the values of $v$ and FWHM recorded using the $632.8 \mathrm{~nm}$ laser are sligthly lower than the one recorded using the $514.5 \mathrm{~nm}$ laser. This may be due to a resonance effect, as suggested previously in [40] and references therein, to explain the slight discrepencies found in the literature.

\section{Discussion}

The comparison of vibrational spectra obtained from quantum calculations of amorphous and crystalline Be including $D$, and Raman spectra obtained for the dendrites formed on the $D$ implanted $\mathrm{Be}$ sample indicates that the $\mathrm{D} / \mathrm{Be}$ ratio in dendrites is close to 2 and the structure is probably crystalline and not amorphous. On the zone of the sample where $10 \%$ of the surface is 
covered by dendrites, the number of $D$ retained in such structures is $N=\left(310^{17}-210^{17} \mathrm{D} \mathrm{cm}{ }^{-2}\right)=1$ $10^{17} \mathrm{D} \mathrm{cm}^{-2}$. Knowing that dendrites are $90 \mathrm{~nm}$ in height and cover $10 \%$ of the surface, the density, expressed in $\mathrm{g} \mathrm{cm}^{-3}$, is:

$\rho=(N \times(2+9 / x)) /\left(5.4 \times 10^{15}\right)$

where $x=\mathrm{D} / \mathrm{Be}$. Assuming a density of $1.23 \mathrm{~g} \mathrm{~cm}^{-3}$, obtained from quantum calculations for the $\mathrm{BeD}_{2}$ crystal (see Table 1), $\mathrm{x}$ is found equal to 2.1 , confirming in very good agreement the assumption of the crystalline structure suggested by the comparison of calculated and measured spectra.

Knowing first that dendrites appear when $N_{D}$ is higher than $210^{17} \mathrm{D} \mathrm{cm}^{-2}$, and second that the 450 and $500 \mathrm{~K}$ TDS peaks of $[13,14]$ (with samples implanted in similar conditions than ours) which appear for fluences higher than $1.210^{17} \mathrm{D} \mathrm{cm}^{-2}$, we propose that the two peaks result from the thermal degradation of dendrites.

The formation of circular domes and dendrites is reported here, but not fully explained. We only suggest that increasing tensile stress in the material (suggested by both Raman measurements and quantum calculations) due to volume increase subsequently to Be-D bond creation may play a role in the formation of the $\mathrm{BeD}_{2}$ crystal. It is also evident that the impinging ion kinetic energy, tuning the implantation depth in the material, is a key parameter in creating this stress, similarly to what was found on bombarded graphite: the height of nanometer sized domes was observe to increase when a higher amount of underlying amorphous carbon (generating stress) was created, using higher implantation energies [44]. In the literature, other structures have been obtained on Be samples after irradiation: cone-like shapes under plasma exposures in the PISCES-B plasma facility [45], interconnected labyrinths with hydrogen bubbles at higher energies [46]. In ITER, only taking into account the charge exchange process leads to anticipate at least a homogeneous $4 \times 10^{17}$ $\mathrm{cm}^{-2} \mathrm{~s}^{-1}$ flux bombarding the Be walls [47]. Extrapolating our result to this flux, we guess that the initial Be surface state in ITER will be completely transformed in a rough surface in only few seconds. These surface modifications under bombardment can significantly influence the local sputtering yield 
[48], which is of importance for extrapolating the lifetime of the future ITER reactor beryllium walls, future work should focus on the understanding of formation mechanisms leading to these structures (and for example explaining the route leading from dome formation to dendrite formation). However, to our knowledge the observation of such dendrites was not yet reported in the JET tokamak with ITER-like wall configuration where such fluences can be found.

The eventual role of particle (sputtered Be, hydrogen,...) mobility on the surface may also be considered for explaining these dendrites formation in the framework of the diffusion limited aggregation model [49]. Stability of the sample after being placed in air for several months (time spent between the different analyses) should also be addressed.

In parallel, but for hydrogen storage purposes, ion assisted techniques should be used, systematically varying the relevant parameters, to optimize the volume of $\mathrm{BeD}_{2}$ formed, to increase the possibility of storing hydrogen isotope in beryllium.

\section{Conclusion}

After deuterium ion irradiation of a polycrystalline beryllium target $(2 \mathrm{keV} / \mathrm{D})$, we have shown that deuterium is trapped up to a saturation value of $\mathrm{D} / \mathrm{Be} \approx 0.24$ when $\mathrm{N}_{\mathrm{D}}=1.510^{17} \mathrm{D} \mathrm{cm} \mathrm{cm}^{-2}$, with an increasing tensile stress developing inside the material, and saturating at this $\mathrm{N}_{\mathrm{D}}$ value. For higher $N_{D}$ values, we have observed that a higher amount of $D$ can be trapped in dendritic structures that start to grow at $N_{D}=2.010^{17} \mathrm{D} \mathrm{cm}^{-2}$ and cover for example $10 \%$ of the surface when $N_{D}=310^{17} \mathrm{D}$ $\mathrm{cm}^{-2}$. By using a multi-technique approach (nuclear reaction analysis, atomic force microscopy, optical microscopy, and quantum calculations compared together with Raman microscopy), we conclude that these dendrites are crystalline and composed of $\mathrm{BeD}_{2}$. These structures can be suitable for storing hydrogen, even if a safety issue concerning the use of Be needs to be performed. As they might play a role in hydrogen isotope retention, future experiments should be devoted to search for such dendrites in the JET tokamak, or in the future ITER tokamak. 


\section{Acknowledgments}

This work has been carried out within the framework of the EUROfusion Consortium and has received funding from the European Union's Horizon 2020 research and innovation program under grant agreement number 633053 . The views and opinions expressed herein do not necessarily reflect those of the European Commission. The authors were granted access to the HPC resources of [CCRT/CINES/IDRIS] under the allocation i2014080509 made by GENCI (Grand Equipement National de Calcul Intensif).

\section{Bibliography}

[1] R. Neu, A.U. Team, E.P. Taskforce, J.E. Contributors, Preparing the scientific basis for an all metal ITER, Plasma Physics and Controlled Fusion, 53 (2011) 124040.

[2] B. Sakintuna, F. Lamari-Darkrim, M. Hirscher, Metal hydride materials for solid hydrogen storage: A review, International Journal of Hydrogen Energy, 32 (2007) 1121-1140.

[3] J. Roth, E. Tsitrone, A. Loarte, Plasma-wall interaction: Important ion induced surface processes and strategy of the EU Task Force, Nuclear Instruments \& Methods in Physics Research Section BBeam Interactions with Materials and Atoms, 258 (2007) 253-263.

[4] J. Roth, E. Tsitrone, A. Loarte, T. Loarer, G. Counsell, R. Neu, V. Philipps, S. Brezinsek, M. Lehnen, P. Coad, C. Grisolia, K. Schmid, K. Krieger, A. Kallenbach, B. Lipschultz, R. Doerner, R. Causey, V. Alimov, W. Shu, O. Ogorodnikova, A. Kirschner, G. Federici, A. Kukushkin, E.P.T. Force, I.P. Team, E. Fusion, D.I.V. Itpa Sol, Recent analysis of key plasma wall interactions issues for ITER, Journal of Nuclear Materials, 390-91 (2009) 1-9.

[5] D. Borodin, S. Brezinsek, J. Miettunen, M. Stamp, A. Kirschner, C. Bjorkas, M. Groth, S. Marsen, C. Silva, S.W. Lisgo, D. Matveev, M. Airila, V. Philipps, J.-E. Contributors, Determination of Be sputtering yields from spectroscopic observations at the JET ITER-like wall based on three-dimensional ERO modelling, Physica Scripta, T159 (2014) 014057.

[6] G. De Temmerman, M.J. Baldwin, R.P. Doerner, D. Nishijima, K. Schmid, An empirical scaling for deuterium retention in co-deposited beryllium layers, Nuclear Fusion, 48 (2008) 75008.

[7] G. De Temmerman, R.P. Doerner, Revised scaling equation for the prediction of tritium retention in beryllium co-deposited layers, Nuclear Fusion, 49 (2009) 042002.

[8] M. Mayer, R. Behrisch, H. Plank, J. Roth, G. Dollinger, C.M. Frey, Codeposition of hydrogen with beryllium, carbon and tungsten, Journal of Nuclear Materials, 230 (1996) 67-73.

[9] R.A. Causey, D.S. Walsh, Codeposition of deuterium with beryllium, Journal of Nuclear Materials, 254 (1998) 84-86.

[10] M.J. Baldwin, K. Schmid, R.P. Doerner, A. Wiltner, R. Seraydarian, C. Linsmeier, Composition and hydrogen isotope retention analysis of co-deposited C/Be layers, Journal of Nuclear Materials, 337 (2005) 590-594.

[11] J.T. Zhao, Q. Wang, T.S. Wang, X.X. Xu, S. Zhang, Y.S. Zhou, X.C. Guan, K.H. Fang, J. Kasagi, Dynamical saturated concentration of deuterium in a beryllium foil studied by low energy $D(d, p) T$ reaction, Nuclear Instruments \& Methods in Physics Research Section B-Beam Interactions with Materials and Atoms, 316 (2013) 13-16.

[12] V.K. Alimov, V.N. Chernikov, A.P. Zakharov, Depth distribution of deuterium atoms and molecules in beryllium implanted with D ions, Journal of Nuclear Materials, 241 (1997) 1047-1051. 
[13] M. Oberkofler, C. Linsmeier, Deuterium release from implanted beryllium and beryllium oxide, Journal of Nuclear Materials, 415 (2011) S724-S727.

[14] M. Reinelt, A. Allouche, M. Oberkofler, C. Linsmeier, Retention mechanisms and binding states of deuterium implanted into beryllium, New Journal of Physics, 11 (2009) 043023.

[15] A.A. Haasz, J.W. Davis, Deuterium retention in beryllium, molybdenum and tungsten at high fluences, Journal of Nuclear Materials, 241 (1997) 1076-1081.

[16] R. Piechoczek, M. Reinelt, M. Oberkofler, A. Allouche, C. Linsmeier, Deuterium trapping and release in Be(0001), Be(11-20) and polycrystalline beryllium, Journal of Nuclear Materials, 438 (2013) S1072-S1075.

[17] M. Fichtner, Nanotechnological aspects in materials for hydrogen storage, Advanced Engineering Materials, 7 (2005) 443-455.

[18] P. Vajeeston, P. Ravindran, H. Fjellvag, Predicting New Materials for Hydrogen Storage Application, Materials, 2 (2009) 2296-2318.

[19] A.W.C. van den Berg, C.O. Arean, Materials for hydrogen storage: current research trends and perspectives, Chemical Communications, (2008) 668-681.

[20] A.D. Zdetsis, M.M. Sigalas, E.N. Koukaras, Ab initio theoretical investigation of beryllium and beryllium hydride nanoparticles and nanocrystals with implications for the corresponding infinite systems, Physical Chemistry Chemical Physics, 16 (2014) 14172-14182.

[21] G.J. Brendel, E.M. Marlett, L.M. Niebylski, CRYSTALLINE BERYLLIUM HYDRIDE, Inorganic Chemistry, 17 (1978) 3589-3592.

[22] M.D. Senin, V.V. Akhachinskii, Y.E. Markushkin, N.A. Chirin, L.M. Kopytin, I.P. Mikhalenko, N.M. Ermolaev, A.V. Zabrodin, THE PRODUCTION, STRUCTURE, AND PROPERTIES OF BERYLLIUM HYDRIDE, Inorganic Materials, 29 (1993) 1416-1420.

[23] E.C. Head, C.E. Holley, S.W. Rabideau, J. Am. Chem. Soc., 79 (1951) 3687.

[24] J.L. Maienschein, J.S. Bowers, J.S. Cantrell, T.A. Beiter, THERMAL CRYSTALLINE-TO-AMORPHOUS TRANSITION OF BERYLLIUM HYDRIDE, Journal of Alloys and Compounds, 179 (1992) 157-163.

[25] C. Pardanaud, Y. Addab, C. Martin, P. Roubin, B. Pegourié, M. Oberkofler, M. Köppen, T. Dittmar, C. Linsmeier, Raman microscopy as a defect microprobe for hydrogen bonding characterization in materials used in fusion applications, physica status solidi (c), 12 (2015) 98-101.

[26] D. Reed, D. Book, Recent applications of Raman spectroscopy to the study of complex hydrides for hydrogen storage, Current Opinion in Solid State \& Materials Science, 15 (2011) 62-72.

[27] C. Linsmeier, P. Goldstrass, K.U. Klages, ARTOSS - A new surface science experiment to study the hydrogen inventory in multi-component materials, Physica Scripta, T94 (2001) 28-33.

[28] M. Oberkofler, C. Linsmeier, Properties of nitrogen-implanted beryllium and its interaction with energetic deuterium, Nuclear Fusion, 50 (2010) 125001.

[29] J.P. Perdew, K. Burke, M. Ernzerhof, Generalized gradient approximation made simple, Physical Review Letters, 77 (1996) 3865-3868.

[30] S. Grimme, Accurate description of van der Waals complexes by density functional theory including empirical corrections, Journal of Computational Chemistry, 25 (2004) 1463-1473.

[31] E.J. Meijer, M. Sprik, A density-functional study of the intermolecular interactions of benzene, Journal of Chemical Physics, 105 (1996) 8684-8689.

[32] P. Giannozzi, S. Baroni, N. Bonini, M. Calandra, R. Car, C. Cavazzoni, D. Ceresoli, G.L. Chiarotti, M. Cococcioni, I. Dabo, A. Dal Corso, S. de Gironcoli, S. Fabris, G. Fratesi, R. Gebauer, U. Gerstmann, C. Gougoussis, A. Kokalj, M. Lazzeri, L. Martin-Samos, N. Marzari, F. Mauri, R. Mazzarello, S. Paolini, A. Pasquarello, L. Paulatto, C. Sbraccia, S. Scandolo, G. Sclauzero, A.P. Seitsonen, A. Smogunov, P. Umari, R.M. Wentzcovitch, QUANTUM ESPRESSO: a modular and open-source software project for quantum simulations of materials, Journal of Physics-Condensed Matter, 21 (2009) 395502.

[33] K.F. Garrity, J.W. Bennett, K.M. Rabe, D. Vanderbilt, Pseudopotentials for high-throughput DFT calculations, Computational Materials Science, 81 (2014) 446-452.

[34] A. Shayesteh, K. Tereszchuk, P.F. Bernath, R. Colin, Infrared emission spectra of BeH2 and BeD2, Journal of Chemical Physics, 118 (2003) 3622-3627. 
[35] J. Koput, K.A. Peterson, Ab initio prediction of the potential energy surface and vibrationrotation energy levels of BeH2, Journal of Chemical Physics, 125 (2006) 044306.

[36] E. Wachowicz, A. Kiejna, Bulk and surface properties of hexagonal-close-packed Be and Mg, Journal of Physics-Condensed Matter, 13 (2001) 10767-10776.

[37] A. Allouche, M. Oberkofler, M. Reinelt, C. Linsmeier, Quantum Modeling of Hydrogen Retention in Beryllium Bulk and Vacancies, Journal of Physical Chemistry C, 114 (2010) 3588-3598.

[38] R. Stedman, Z. Amilius, R. Pauli, O. Sundin, PHONON SPECTRUM OF BERYLLIUM AT $80 \mathrm{~K}$, Journal of Physics F-Metal Physics, 6 (1976) 157-166.

[39] G. Gouadec, P. Colomban, Raman spectroscopy of nanomaterials: How spectra relate to disorder, particle size and mechanical properties, Progress in Crystal Growth and Characterization of Materials, 53 (2007) 1-56.

[40] W.J. Evans, M.J. Lipp, H. Cynn, C.S. Yoo, M. Somayazulu, D. Hausermann, G. Shen, V. Prakapenka, $X$-ray diffraction and Raman studies of beryllium: Static and elastic properties at high pressures, Physical Review B, 72 (2005) 094113.

[41] G. Morell, W. Perez, E. ChingPrado, R.S. Katiyar, Anharmonic interactions in beryllium oxide, Physical Review B, 53 (1996) 5388-5395.

[42] S. Sampath, A.I. Kolesnikov, K.M. Lantzky, J.L. Yarger, Vibrational dynamics of amorphous beryllium hydride and lithium beryllium hydrides, Journal of Chemical Physics, 128 (2008).

[43] J.F. Ziegler, J.P. Biersack, U. Littmark, The Stopping and Range of lons in Matter, Pergamon: New York, 1995.

[44] C. Pardanaud, C. Martin, G. Cartry, A. Ahmad, L. Schiesko, G. Giacometti, M. Carrere, P. Roubin, In-plane and out-of-plane defects of graphite bombarded by $\mathrm{H}, \mathrm{D}$ and He investigated by atomic force and Raman microscopies, Journal of Raman Spectroscopy, 46 (2014) 256-265.

[45] R.P. Doerner, D. Nishijima, T. Schwarz-Selinger, Impact of surface morphology on sputtering during high-fluence plasma exposure, Physica Scripta, T159 (2014) 014040.

[46] V.N. Chernikov, V.K. Alimov, A.V. Markin, A.E. Gorodetsky, S.L. Kanashenko, A.P. Zakharov, I.B. Kupriyanov, Gas-induced swelling of beryllium implanted with deuterium ions, Journal of Nuclear Materials, 233 (1996) 860-864.

[47] S. Carpentier, R.A. Pitts, P.C. Stangeby, J.D. Elder, A.S. Kukushkin, S. Lisgo, W. Fundamenski, D. Moulton, Modelling of beryllium erosion-redeposition on ITER first wall panels, Journal of Nuclear Materials, 415 (2011) S165-S169.

[48] R.P. Doerner, C. Bjoerkas, D. Nishijima, T. Schwarz-Selinger, Erosion of beryllium under high-flux plasma impact, Journal of Nuclear Materials, 438 (2013) S272-S275.

[49] T.A. Witten, L.M. Sander, Diffusion-limited aggregation, Physical Review B, 27 (1983) 5686-5697. 


\begin{tabular}{|c|c|c|}
\hline \multicolumn{2}{|c|}{ D/Be } & $\begin{array}{c}\text { Density } \\
\left(\text { g.cm }^{-3}\right)\end{array}$ \\
\hline \multirow{3}{*}{ Amorphous } & 0 & 1.95 \\
\cline { 2 - 3 } & 0.5 & 1.77 \\
\cline { 2 - 3 } & 1 & 1.58 \\
\cline { 2 - 3 } & 2 & 1.33 \\
\hline Crystal & 2 & 1.23 \\
\hline
\end{tabular}

Table 1. Volume and density evolution as a function of deuterium concentration at the pressure of 1 atmosphere.



Figure 1. Calculated vibrational frequencies obtained in dressing the numerical values by a Gaussian function of the $\mathrm{BeD}_{2}$ molecule in beryllium and in gas phase (same convention in Figures 2 and 4). 


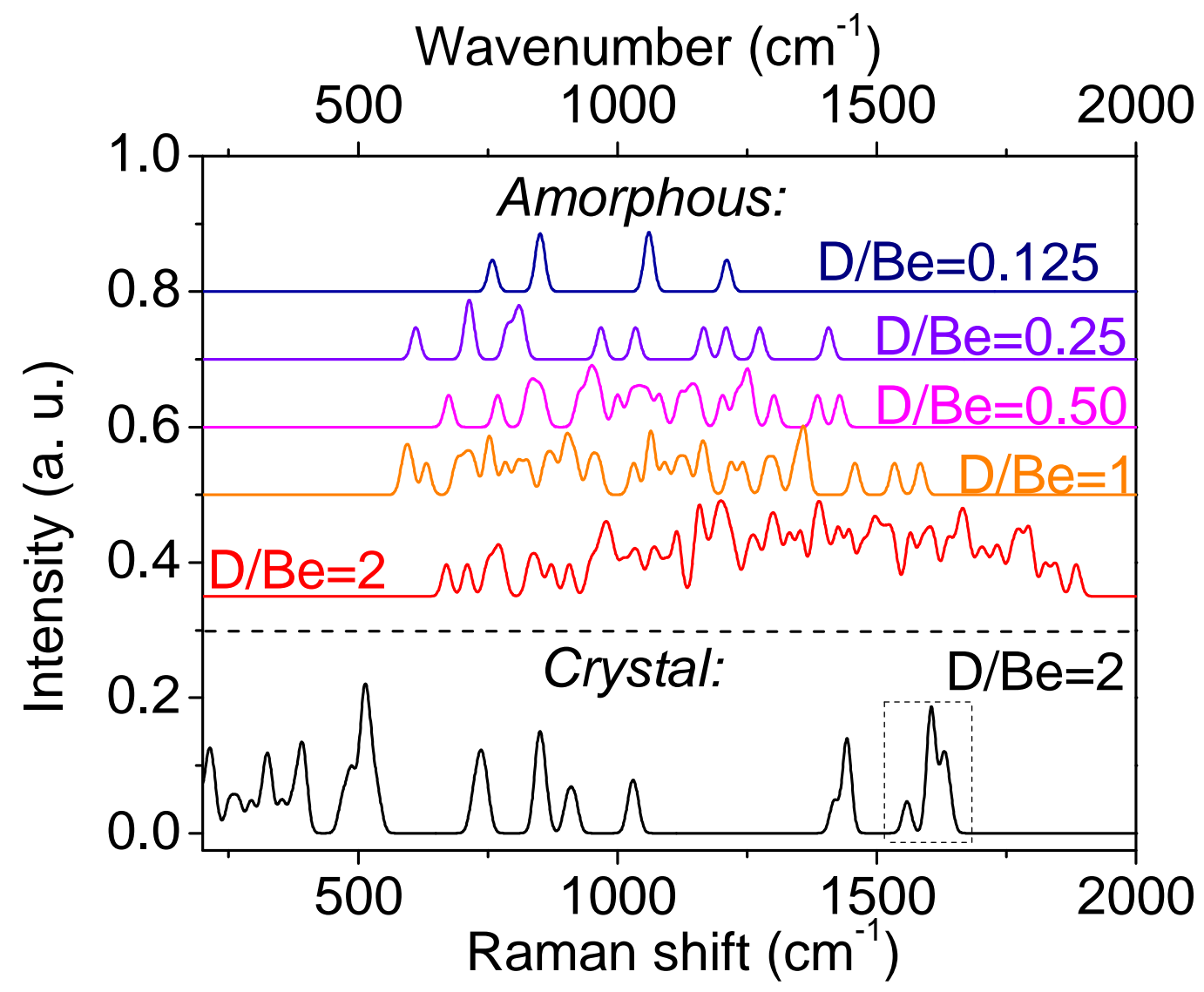

Figure 2. Vibrational frequencies associated to deuterium trapping in the beryllium crystal as a function of the deuterium concentration ( $D / B e$ atomic ratio), only the modes involving $D$ are represented. In the lower part of the figure the calculated full beryllium hydride crystal Raman active frequencies are shown. 


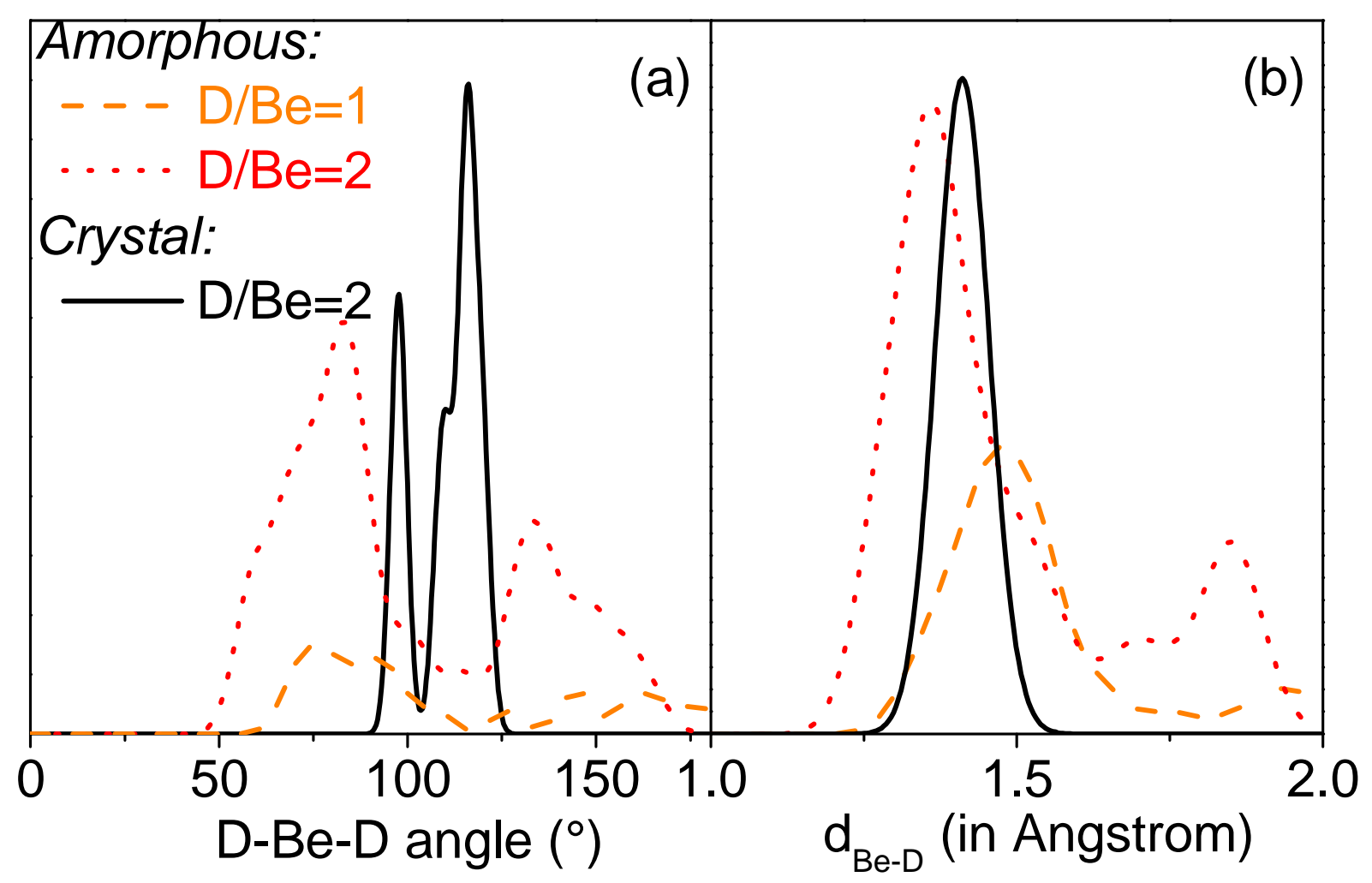

Figure 3. Beryllium hydride structure evolution towards a tetrahedral beryllium one, comparison with the beryllium hydride crystal. (a) Angular distribution in amorphous and ordered beryllium hydrides. (b) Be-D length distribution in amorphous and organized beryllium hydrides. Only the Be-D distances shorter than $2 \AA$ are considered. 


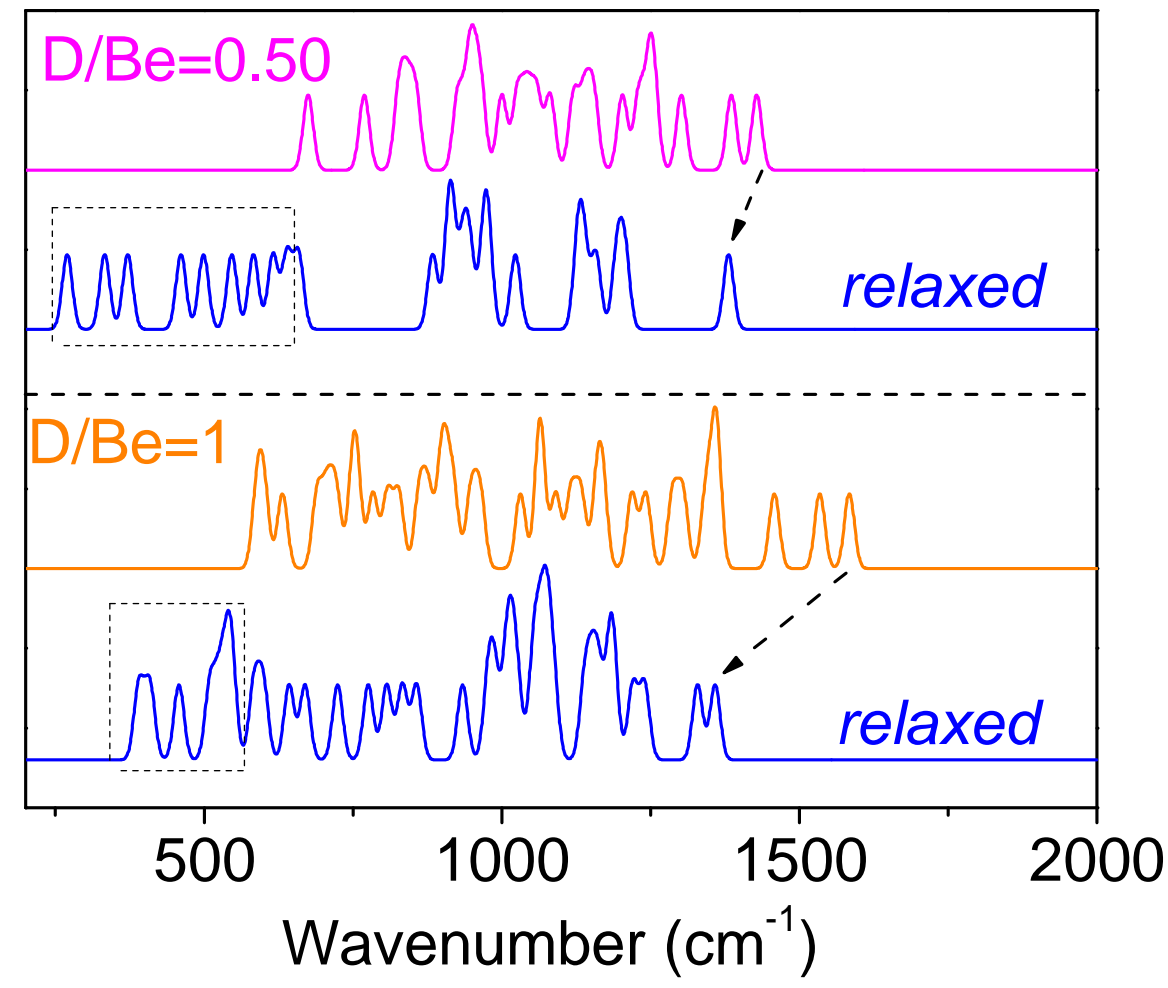

Figure 4. Vibrational frequencies for the ratio $\mathrm{D} / \mathrm{Be}=0.5$ (a) and 1.0 (b) after cell parameter relaxation, compared to the original beryllium cell. 




Figure 5. Raman spectra of deuterium implanted beryllium. (a) Beryllium stretching mode spectral window. (b) Beryllium deuteride spectral window. The lowest Raman spectrum is the calculated $\mathrm{BeD}_{2}$ crystal. For each spectrum, the colors indicate the $\mathrm{D}$ areal densities. 

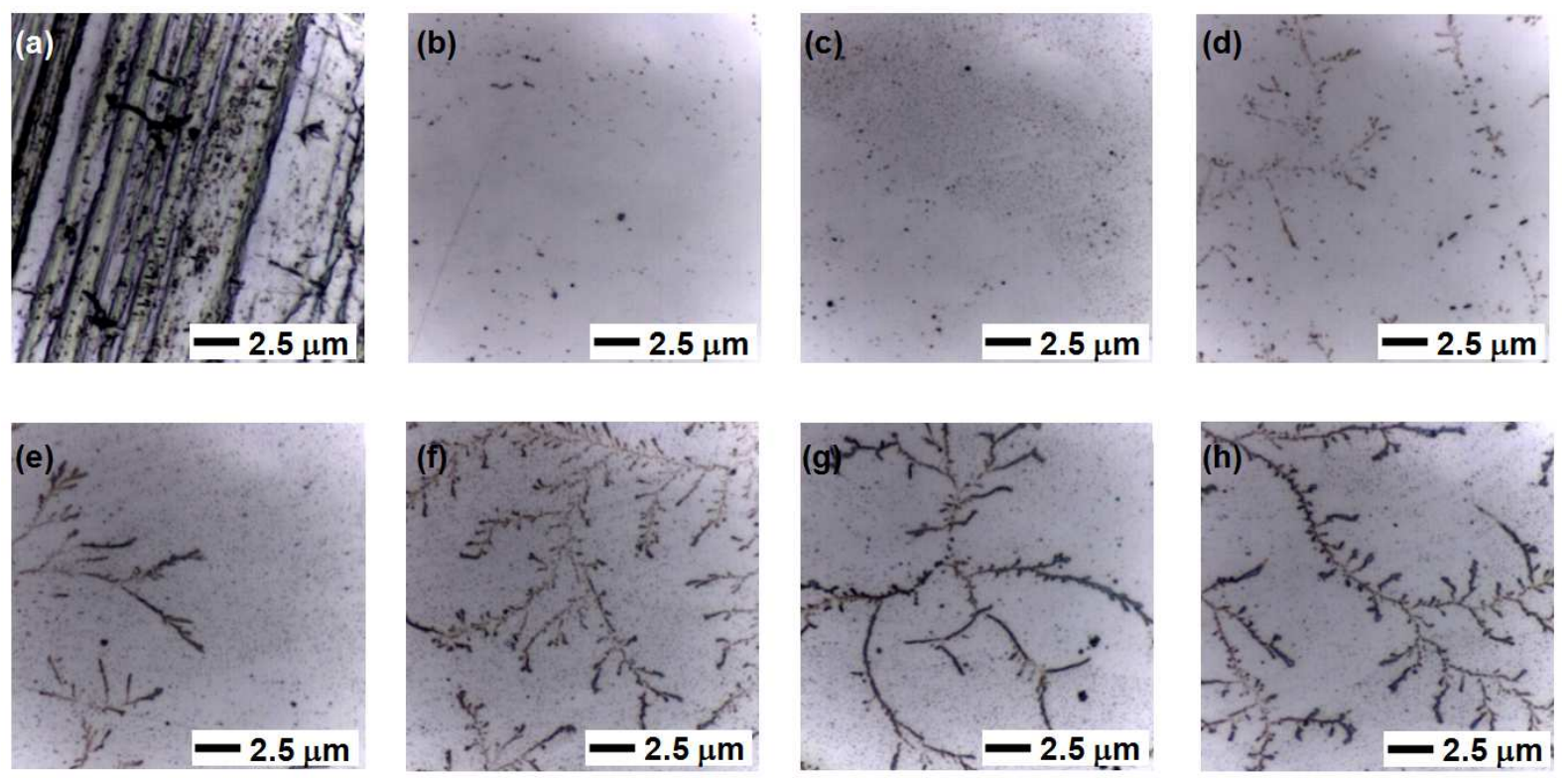

Figure 6. Optical microscope images of various regions on a deuterium-implanted beryllium sample.

(a) Pristine. (b) and (c) In zones of the sample where $N_{D}<2.010^{17} \mathrm{D} \mathrm{cm}^{-2}$. (d) to (g) In zones of the sample where $\mathrm{N}_{\mathrm{D}}>2.010^{17} \mathrm{D} \mathrm{cm}^{-2}$. 




Figure 7. Height of the deuterium implanted sample in zones containing $N_{D}>2.010^{17} \mathrm{~cm}^{-2}$, obtained by AFM. (a) and (b) represent typical morphologies found in that zone. 

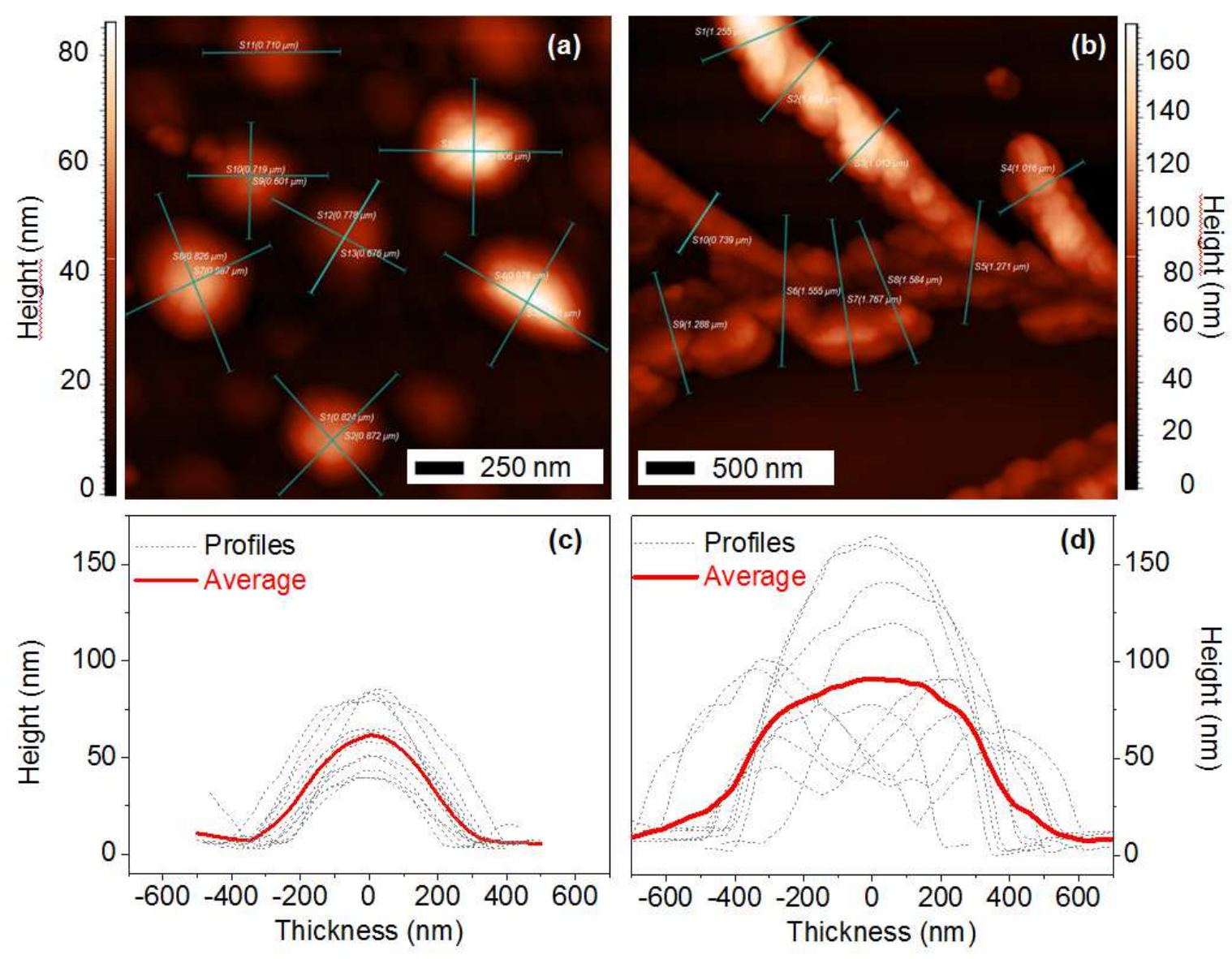

Figure 8. Height profile analysis of the domes and dendrites. (a) and (b) are AFM images representing the height of domes and dendrites. (c) and (d) show profiles extracted from (a) and (b) respectively. 

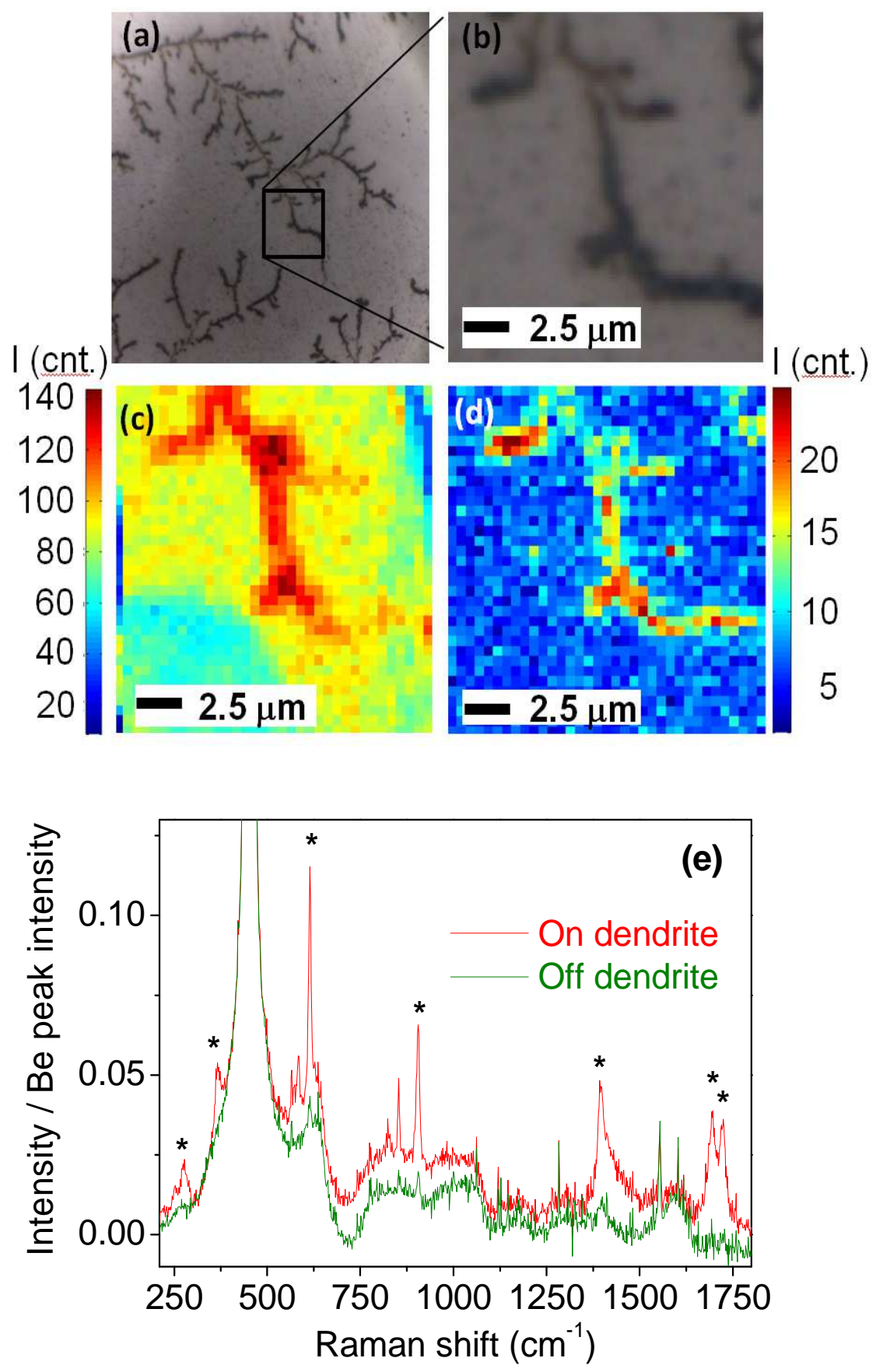

Figure 9. Raman imaging in the vicinity of a dendrite. (a, b) optical microscope images. (c) Intensity of the band corresponding to the Be stretching mode. (d) Intensity of the band corresponding to the mode lying at $1397 \mathrm{~cm}^{-1}$, in the same intensity unit as in (c). The zone on the sample corresponds to $N_{D}=310^{17} \mathrm{D} \mathrm{cm}^{-2}$. (e) Comparison of on-dendrite and off-dendrite Raman spectra. Spectra were summed over the two areas and are then normalized to the beryllium band, fixed at a value of 1 . The threshold for distinguishing if a zone is on or off dendrite was fixed at a value of 100 in Figure 9-c. 




Figure 10. Evolution of Raman parameters and dendrite surface coverage versus the quantity of implanted deuterium. (a) and (b) Be stretching mode band center and full-width-at-half-maximum, respectively. (c) Surface coverage of the dendrites retrieved by optical microscopy. 